\title{
La casa de los espejos: Las élites de Barcelona y las transiciones alfonsinas
}

\begin{abstract}
GARI W. MCDONOGH
Els homes responsables de totes les desgracies d'Espanya segueixen contra nosaltres aquesta politica de divisió social, volent provocar entre nosaltres la guerra de classes (La Veu de Catalunya 2-III-1902).

En aquesta campanya contra la Lliga coincideixin, amb coincidencia sospitosa diaris comunistes com Solidaridad Obrera i diaris burgesos com El Imparcial i representants autentics de la vella política madrilenya que el cop d'estat enderroca, $L a$ Libertad i l'ABC (La Veu de Catalunya 22-IX-1923).
\end{abstract}

El 2 de mayo de 1888, el rey de España Alfonso XIII, de dos años de edad, rodeado por su familia, la reina regente, representantes de las casas reales de Europa y el presidente del consejo de Ministros, Sagasta, con los miembros del gobierno español, inauguró la Exposición Universal de Barcelona de 1888. Cuarenta años después, en 1929, el rey volvió a Barcelona para la inauguración de la segunda exposición internacional patrocinada por la ciudad. Esta exposición se cerró el 15 de enero de 1930, pocos días antes de la renuncia del General Primo de Rivera de sus poderes dictatoriales, y un año antes de la caída y del exilio del propio rey $y$ el nacimiento de la segunda república española.

Estas dos exposiciones, llenas de significados económicos, políticos, sociales y culturales para la élite industrial-financiera de la ciudad, ofrecen una simetría perfecta para el estudio de los grupos que componían la sociedad barcelonesa durante la época alfonsina. Sin embargo, es un paralelismo falso, cuya desigualdad nos indica las contradicciones fundamentales de la formación de la élite en estas décadas. Una exposición en 1887 fue propuesta en 1883 por Eugenio R. Serrano de Casanova, un empresario gallego residente en París; quería una representación del Estado español, ubicada en su centro industrial, Barcelona ${ }^{1}$. Con la reno-

Existía, sin embargo, una arraigada tradición de exposiciones industriales en Barcelona, 
vación política que siguió a la muerte de Alfonso XII, el nuevo alcalde de Barcelona, Francesc de P. Rius i Taulet, ofreció ayuda municipal al proyecto. Un año antes de la fecha discutida para la inauguración, cuando se veía que Serrano no podía completar su programa, Rius i Taulet selló una alianza de prohombres urbanos procedentes de la política, del mundo académico, del banco y de la industria, con la ayuda fiscal de las Cortes. Aunque algunos críticos catalanistas, notablemente Valentí Almirall, rechazaron el proyecto, un considerable esfuerzo urbanístico completó toda una serie de edificios y atracciones con que recibir a los visitantes extranjeros y locales en 1888. Uno de los Congresos complementarios celebrados en la ciudad en este año fue la primera reunión de la U.G.T. (Unión General de Trabajadores) y el P.S.O.E. (Partido Socialista Obrero Español).

La Exposición Internacional de 1929 tuvo una historia más complicada. Rius i Taulet, al cerrar la primera exposición propuso una segunda, un desafio que llegó a ser «un punt de referencia per als diversos consistoris municipals i per la societat urbana en general» (GRAU 1988: 369). Discutido en serio en 1906, después de la guerra ultramarina y los triunfos y desastres parlamentarios de los partidos catalanistas, el proyecto se desarrollaba con lentitud a causa del ambiente político polarizado del principado. El centro de la exposición llegó a ser la electricidad, fuerza motriz de la segundo revolución industrial. El gran arquitecto-nacionalista Josep Puig i Cadafalch y la Lliga comenzaron anteproyectos en el 1914 para una inauguración en 1917, fecha que coincidió con la Primera Guerra Mundial y una crisis local. Así, intervenían en la génesis de la exposición huelgas, las elecciones de alcaldes posdinásticos, los años sangrientos de la posguerra. Puig, como presidente de la Mancomunitat de Catalunya, continuaba las obras. Con el colapso del gobierno central y la entrada en el poder de Primo de Rivera con el espaldarazo de la élite barcelonesa, Puig y la Lliga perdieron primero su mando político y después su autoridad arquitectónica sobre los campos de Montjüic. La exposición se inauguró al final en un ambiente teñido de mediocridad arquitectónica e incertidumbre política ${ }^{2}$.

incluso discusión de anteproyectos dentro del Ateneu Barcelonés (Grau 1988: 316-319). El centenario de la exposición ha producido estudios fundamentales de la feria y su época, incluso las de Grau 1988 y Heuret 1988.

2 Entre las obras fundamentales para el estudio de la Exposición de 1929 se encuentran Sola Morales (1976); Grandas (1988) y Barcelona y sus Exposiciones (1929). Además, debemos reconocer estudios fundamentales del urbanismo, crisis y planificación sobre esta época: Torres Capell (1987); Martorell et al (1970); Rovira Gimeno (1987); Bomigas (1968), etc. 
Las relaciones político-económicas con el gobierno central que se ven en el desarrollo de estas dos exposiciones constituyen un elemento de la imagen de los "espejos" que aparece en el título de esta ponencia. Las clases dominantes de Barcelona en la época de Alfonso XIII reflejaban las aspiraciones y preocupaciones del régimen dinástico, así como el potencial de crecimiento industrial y cultural de Catalunya que podía haber sostenido una España europea y alternativa dentro de la revolución industrial. 1888 representaba un desafío mientras que la exposición de 1929 aceptaba unas limitaciones más conservadoras. Sin embargo, otro nexo de conflicto determinaba igualmente el desarrollo de esta élite de una manera que se puede distinguir de otras élites españolas. Las clases dominantes se consolidaron en Barcelona en el seno de un conflicto con otros grupos sociales que brotaron de la misma revolución industrial. El conflicto entre dueño y obrero, capitalistas y proletariado, y las estrategias hegemónicas que entrelazaron a los capitalistas con otros fragmentos de clase de la burguesía y los gerentes de la ciudad, ofrecía otro espejo a la cara de la alta burguesía barcelonesa. En el siglo XIX vemos la organización y desarrollo de conciencia de la clase obrera, señalada entre otras cosas por la formación de la U.G.T. En el siglo xx, se nota la representación pública del poder del proletariado en el ayuntamiento y en las calles en 1909 y en la década siguiente ${ }^{3}$. Además, otras voces, como Francesc Macià y Esquerra Catalana, contestaron al poder alto-burgués de la Lliga. La necesidad de la primera élite alfonsina era dominar dentro de Catalunya y a la vez adquirir y manipular poder dentro del estado español. Entre estos dos espejos de poder yacían unas contradicciones fundamentales que situaban a estas familias en una ambigüedad destructiva.

En este ensayo, quiero plantear los efectos sociales y culturales de estas contradicciones, la tierra entre los espejos deformantes. Mi formación antropológica me ha enfocado hacia temas de la representación cultural del poder, dentro de los modelos propuestos por Abner COHEN (1969, 1974 y 1981) y Pierre Bourdieu $(1972,1977)$, en vez de un análisis de sucesos políticos o económicos de la época. Considero, en una frase clave del primero, que "social anthropology is essentially concerned with the dialectical relation between two major variables: symbolic action and political relationships (1974:21). Así, quiero reflexionar sobre estas contradicciones en sus manifestaciones dentro del tema de la formación fa-

3 Hay una bibliografía complicada sobre la organización obrera en Catalunya (Véase nota 5). Culla I Clara (1986) ha hecho un estudio detallado del fenómeno de Lerroux dentro de este contexto político. 
miliar de la élite que empecé a investigar hace quince años, considerando ahora a la familia como límite dentro de un contexto sincrónico en vez de un proceso. Siguen consideraciones que han surgido de investigaciones sobre clase y categoría como estrategia que corresponde al desarrollo histórico de un simbolismo urbano de distinción, en el modelo bourdieuano, y sobre arquitectura urbana y las fachadas de poder. Estas reflexiones interdisciplinarias complementarán nuestras discusiones y nuestra comprensión de unos procesos claves en la formación de la Barcelona y la España modernas.

\section{LA FAMILIA ENTRE ORGANIZACIÓN Y SIMBOLO}

Como ya he señalado en el estudio sobre la formación de las «Buenas Familias" de Barcelona de la época industrial, la familia tenía un papel central dentro de la organización y la reproducción de esta élite en el siglo xix y en su proyección cultural en Catalunya y España (McDoNOGH 1986). La familia, envuelta en una red de contextos emocionales e históricos, ofrecía una estructura y un simbolismo ideal para la unificación y representación de antiguas y nuevas clases poderosas. La representación de Catalunya como familia afirmaba su unidad nacional ante el estado central. Como otros nacionalistas europeos del siglo xIX, los barceloneses llegaron a la metaforización de la familia, raza, sangre y nación como entidades trascendentes dentro de las cuales podían construir la estructura política de su nacionalidad ${ }^{4}$. A la vez, el modelo patriarcal de la casa tradicional-reinventada ofrecía pautas emocionales para la interpretación mítica de relaciones de clase dentro de la fábrica, la ciudad y el estado. Los dueños se vieron como padres aunque sus «hijos» obreros no aceptasen el rol que los patrones les ofrecían (MCDONOGH 1986; TERRADES $1978,1979)^{5}$.

En cuanto al uso social de la familia dentro de la gerencia y la organización legal de la empresa, por ejemplo, el fenómeno más característico de la época alfonsina era la transformación de la sociedad colectiva en una sociedad anónima de índole familiar. Repasando datos del registro

\footnotetext{
4 El tema de raza aparece en MARFany (1978). También, este tema ha surgido en la discusión con Verena Stolke (com. pers.).

5 El rechazo violento de esta imagen, tanto como su aceptación dentro de otros segmentos de la clase obrera cae fuera de este ensayo. Debo mencionar en este aspecto el trabajo importante de Ignasi TerRades Saborit $(1978,1979)$, Miquel IzARo $(1973,1979)$ y otros historiadores y antropólogos de la clase obrera catalana que me han ofrecido una pista que todavia no he podido desarrollar.
} 
mercantil, de libros memoriales de la industria textil y de historias familiares, se ve un florecimiento extraordinario de empresas de esta categoría en las primeras décadas del siglo xx. Mientras que en los años 1890-1909, sólo aparecieron dos fundaciones de este tipo, entre 1910 y 1919 se inscribieron diez, y entre 1920 y 1929, treinta (MCDONOGH 1988:103-105; EsPIRITU Y FUERZA 1945). Este patrón ha sido confirmado por un modelo de tres generaciones a través del cual las clases dominantes han interpretado su propia historia: el padre que fundó la empresa, el hijo que la desarrolló y los nietos que la perdieron (VICENS VIVES 1958). En este mito, la generación de los nietos - de pérdida y de inseguridad-correspondía en parte a las primeras décadas del siglo xx. Pero entre las causas inmediatas de la transformación, debemos incluir la resistencia de la clase obrera, que tomaba una forma cada vez más organizada y aún violenta después de la Setmana Tràgica de 1909, la crisis de 1917 y la inseguridad del mercado internacional después de la Primera Guerra Mundial (McDonOGH 1988:84-109, 268-274; TorRes CAPELl 1987).

La mentalidad de crisis apareció en el periodismo barcelonés antes del golpe de Estado de Primo de Rivera.

\footnotetext{
En Barcelona, huelgas, atracos, robos; en Madrid... sigue la farsa política. (Correo Catalán 10-VII-1923).

Un regim que era una ficció : una vergonya, que era una clientela i una tertulia, que era l'arbitrarietat $i$ el convencionalismo i del quals tots els pobles espanyols, i molt especialment Catalunya, no havien rebut sinó greuges.

La caiguda de la concentració lliberal i del sistema polític al qual pertanyia ha estat rebuda per tot el pais amb aplaudiment unanime (La Veu de Catalunya 17-IX-1923).
}

La transformación de mentalidad subraya un habitus de seguridad que contrasta con la formación anterior de negocios y grupos financieros de un carácter estatal o internacional, representada por el imperio empresarial fundado por Antonio López y López, primer Marqués de Comillas. López construyó una red de parentesco e influencia que se extendía de Barcelona y Santander a Madrid y el País Vasco. Este uso potente de la forma de la sociedad anónima fue desarrollado después de su muerte por su hijo, Claudio, quien se aprovechó de las oportunidades y necesidades de la segunda revolución industrial, así como de las oportunidades sociales del veraneo en Comillas, hasta su muerte en 1925. La tercera generación, sin embargo, "mantenía" el estatus quo en vez de adaptar o hacer crecer. Sólo emergieron algunas empresas de explotación urbanística que solían surgir de propiedades familiares desarrollados a través de sociedades anónimas familiares (MCDONOGH 1988:113-117; 125-133; 280- 
287). Mientras tanto, las nuevas grandes empresas de la época, como el CHADE (Compañía Hispano Americana de Electricidad), muestran la influencia de un grupo gerente-político, epitomado por Francesc Cambó y sus relaciones con Madrid y el exterior (PABÓN 1952-1969, MCDONOGH: 1986 135-137).

La formación de la empresa familiar representaba una lógica que valoriza la familia, la reproducción, y la seguridad. Así, podemos localizarlo dentro de un proceso histórico. A la vez, podemos reconocer que esta transformación evitaba riesgo, pero también posibilidades de cualquier crecimiento importante. Su lógica generacional-procesal, mitificada por los descendientes del empresario fundador, puede disfrazar un límite sincrónico importante. Podemos calificar a estas disposiciones y estrategias que respondían a la inseguridad dual de la situación como un habitus, en el sistema de Bordieu ${ }^{6}$.

Repasando otros datos de la formación y representación de la familia en esta época se notan ecos del mismo habitus conservador. En el mercado del capital social representado por los matrimonios de las familias pudientes de las generaciones alfonsinas, por ejemplo, la élite tenía una fuerte tendencia a la involución matrimonial. Los matrimonios consolidaban y reconsolidaban familias procedentes de grupos anteriormente en competencia con la alta burguesía - comercial, financiera e industrial- y la nobleza terrateniente. Incorporaron unos pocos gerentes a pesar de desniveles complicados en el mercado nupcial, pero los entrelazos matrimoniales apenas extienden hacia Madrid, Bilbao o París. Una endogamia de clase tanto como de región indica otra vez una situación limitante de nexos sociales tanto como de fuerza. ${ }^{7}$

En la representación arquitectónica/simbólica de la élite dentro de la ciudad se nota también la preferencia hacia la reproducción social en vez de nueva construcción. Los grandes monumentos novecentistas de la representación de familia y poder patrocinados por esta clase -el cementiri vell y el Gran Teatre del Liceu - perduraban con la legitimación añadida de la edad (MCDONOGH 1986). Las grandes construcciones urbanas de los años 10 y 20 eran públicas, como las escuelas de Josep Goday que

6 Bourdieu usa habitus para la comprensión de un grupo de disposiciones y estrategias sociales que forma la mentalidad de un grupo en cualquier situación socio-histórica concreta que puede llegar a determinar su actuación en el mundo (1972). Asi entrelaza situación y conciencia dentro de fragmentos de una sociedad compleja.

7 Es evidente que conflictos y competencia surgian entre familias y compañias en el mundo barcelonés. Sin embargo, los matrimonios definian y controlaban algunos aspectos del conflicto económico. 
trataremos posteriormente, o de un modelo de clase distinto, como el templo expiatorio de la Sagrada Familia. Los esfuerzos de urbanización de la élite eran más bien de huida, indicada por el desarrollo suburbano del Parc Güell, el Tibidabo o los campos de Pedralbes. Por lo general, la planificación se convertía en burocracia en vez de campo de intervención patronal (TORRES CAPELL 1987).

Todas estas disposiciones y acciones formaban un habitus que podemos relacionar con la situación contradictoria de la élite, entre el problema del dominio en Catalunya y el de la representación ante Madrid. Las generaciones alfonsinas de la élite financiera-industrial barcelonesa del $x x$, después de una época de enfrentamiento, aceptaron - icrearon?un habitus basado en la reproducción y consolidación. Para comprender mejor el contexto y la respuesta, sin embargo, tenemos que mirar la actuación y la actitud de esta élite en otros campos.

\section{CAPITAL SOCIAL Y CAPITAL CULTURAL}

La transición desde una aristocracia terrateniente a una burguesía emergente del siglo XIX permeaba todos los aspectos de la cultura urbana. La normalidad de un sistema de clasificación de la cultura urbana preindustrial, sea el significado de los títulos nobiliarios o la ceremonia pública, entraba en juego. Al final, las nuevas burguesías adoptaban muchas facetas del estilo y mando aristocrático, convirtiendo capital económico en capital social (BouRdieu 1972, 1977; McDonogh 1986). En este proceso, la formación de una élite incluía una transformación de las categorías significativas de la sociedad barcelonesa.

Esta categorización, que representa la base de la cultura urbana y el conflicto entre ciudadanos, no se ocupaba solamente de la identificación de clases conflictivas, sino de toda una escala de intereses y conciencia social. Es decir, mientras que las clases poderosas intentaban identificar y gobernar a la clase obrera, intentaban "convencer" a clases aliadas. Antonio Gramscl, en sus Cuadernos de la Cárcel, sugería una división entre hegemonia y dominio forzado: "Una clase domina de dos maneras, es decir, domina y gobierna. Gobierna las clases aliadas y domina las clases opuestas" (I:57). Según la lectura gramsciana de Chantal MoufFE (1979; 1989), podemos interpretar la hegemonía como un proceso sintético de los intereses de varias clases. Esta síntesis no implica una homogeneidad, sino una avenencia basada en redes metafóricas. Grupos distintos pueden asentir a la categorización mientras que mantienen sus diferentes valores en cuanto a símbolos claves; podían, últimamente, con- 
traponer «la caseta i l'hortet» a la vida palaciega (DIGIACOMO 1987; véase RuBIO I TUdURI 1933).

Una de las clases aliadas pero diferenciadas de la Barcelona alfonsina, era la pequeña burguesía, la clase del origen histórico de muchas familias de la élite. En los años 20 y 30 , esta clase sostenía uno de los partidos que más desafiaban a la gran burguesía. Examinamos las ambigüedades de alianza y diferenciación entre estas dos categorias creadas de una cultura urbana a través de los textos que socializaban a los jóvenes de los dos grupos (McDonogh. En preparación).

Un catecismo anónimo fechado en 1928, Cartilla Moderna de Urbanidad, nos ofrece una imagen de clase y conducta de las burguesías de su época. Este libro emplea una presentación gráfica de contrastes paralelos entre «la niña bien educada» y «la niña mal educada». Se nota la creación cotidiana de un estereotipo que corresponde a un mundo de símbolos literarios nacionales-clasistas como el "Senyor Esteve" de Santiago Russinyol o la «Teresa» noucentista de Eugeni D'ORS (RussinYoL 1907, 1917; D'Ors 1906).

En su introducción, el libro presenta la educación como riqueza social confirmada por la iglesia y la corte medieval, un capital de clase en el sentido de una sabiduría creada y consagrada por el poder (BOURDIEU 1977). Después, sigue la vida cotidiana y semanal de una estudiante, contrastando la buena con la mala. El autor anónimo presenta a la niña bien educada como obediente, caritativa y religiosa. Mantiene una conciencia de clase y de sus obligaciones. Así durante su paseo, "da paso a personas de respeto" y muestra buenos sentimientos con los menos afortunados. También emplea «con discreción y exactitud el saludo apropiado y adecuado para cada situación". La niña mal educada se nota por su paso extravagante y presumida, su ruido y falta de organización.

Existen contrastes paralelos en el campo de caridad y modestia. La niña bien educada ofrece caridad "verdadera y efectiva, ayudando a veces con dinero, a veces con su propia labor». La caridad ufana es pecado, reflejo del pecado de la niña mala, que se entristece pensando en las que tienen más inteligencia o reciben más fama. La envidia aparece como pecado, mientras que la condescendencia, su inversión dentro de las relaciones de clase, recibe elogio. Para ilustrar estos valores, el libro anota que a la niña bien educada "le gusta dar limosnas y sabe ser generosa». La niña mal educada "trata a la criada con despotismo y es cruel con los animales". La asimetría tiene importancia: la niña buena se muestra educada a través de una larga distancia social (con un mendigo) y generosa con otras niñas casi iguales; la otra repite estos ejemplos con un perro como su símbolo de distancia y la criada como igual. En cuanto a la 
modestia, una niña buena no tiene vergüenza de sus padres «aunque sean de condiciones humildes" y ofrece ayuda a los menos afortunados. La otra se caracteriza por su orgullo, inseguridad y apariencia. La buena mira con claridad a las de abajo; la mala mira con envida a las de arriba.

El epílogo concluye, "pobre o rico, todos los mortales ante Dios, todos son iguales». El libro parece confirmar la idea de un código común -para todas las que tenían acceso a una educación institucional, un privilegio burgués-. Sin embargo, repite constantemente que el acceso ni es igual ni debe serlo. Entre dos burgueses hay siempre una distinción creada, representada. Dentro de estos valores, la bondad aparece en la aceptación de clase y cultura, humilde o rica. La urbanidad exige obligación con las inferiores; las niñas malas intentan imitar a sus superiores, sobre todo las que tienen la inseguridad de caer cerca de las hijas del poder. El contenido de la educación invita a las clases educadas pequeño-burguesas a juntarse y a participar en el estatus y poder de la élite -pero a base de un acuerdo común sobre la distinción social-.

Al educar a las niñas en la cultura y en las categorías de su sociedad, elementos de una élite involucionada insistieron en un modelo basado en una representación compartida ${ }^{8}$. La recreación de categorías fue una estrategia de alianza que chocaba contra otros modelos de la pequeña burguesía, los fragmentos de clase (WRIGHT 1978) que ganaron a la Lliga en las elecciones de 1931. Pero la representación y ambigüedad de un habitus que hemos analizado en las aulas entró en debate público a través de la arquitectura urbana como discurso de poder.

\section{RECONSTRUYENDO BARCELONA: ARQUITECTURA Y PODER}

Una de las imposiciones más importantes de las nuevas clases poderosas de Barcelona en el siglo xix era la transformación de la ciudad misma. El Ensanche ochocentists de Barcelona correspondía a una visión del progreso y urbanismo sintetizado en la Exposición de 1888. La Exposición de 1929 tenía otros efectos urbanísticos, quizás más modestos, como el desarrollo de Montjuic, los barrios obreros adyacentes, y la expansión de la ciudad por los campos de Pedralbes (GRANDAS 1988; SolÁMORALES 1976). En su reforma de la ciudad, tanto como en obras individuales patrocinadas por la élite, vemos otra vez las contradicciones de poder de la Barcelona alfonsina.

\footnotetext{
8 El modelo reaparece en literatura y sociología de clases populares (McDONOGH 1987).
} 
El arquitecto historiador David MACKAY ha sugerido cuatro fases de expresión arquitectónica entre 1854 y 1939: (1) los primeros románticos: la búsqueda de la identidad en el pasado; (2) los románticos modernistas y la búsqueda de la identidad en el presente; (3) los románticos idealistas y la búsqueda de la identidad institucional; y (4) los utópicos modernistas y la búsqueda de identidad en el porvenir (1989). La primera fase cae fuera de nuestra discusión, dentro de las primeras décadas de la revolución industrial y los tratos iniciales entre negociantes de capital económico y aristócratas con poder sobre tiempo (BOURDIEU 1977) ${ }^{9}$. La segunda fase coincidía con la restauración y la Exposición de 1888. En la tercera fase, a principios del siglo $x x$, se ve otro movimiento importante para la comprensión del poder: el noucentisme. La última época, el GATCPAC (Grup d'Arquitectes i Tècnics per al Progrés de l'Arquitectura Contemporània), contrasta con la exposición del 1929. Tenemos que examinar estos movimientos en el contexto de las élites y su representación urbana.

El modernismo puede ser el momento mejor conocido de la arquitectura barcelonesa, un estilo que caracteriza a toda una ciudad (MENDOzA Y MENDOZA 1987, BoHIGAS 1968). Entre los arquitectos y sus patrones se producía un cambio en la fisonomía de Barcelona a finales del siglo XIX, con una fuerte interpenetración de raíces económicas, artísticas y políticonacionalistas. Lluís Domenech i Muntaner y Josep Puig i Cadafalch eran arquitectos y políticos; el segundo, presidente de la Mancomunitat de Catalunya e hijo político de Dolors Monserda, novelista de la nueva burguesía catalana (Bohigas, 1973; Colegio Oficial de ARquitectos 1981). Enric Sagnier Villavecchia, arquitecto del Palacio de Justicia, era descendiente de dos familias comerciales de la élite. Industriales como los López y los Güell, mecenas de Antoni Gaudí, los Milà (del mismo) y el Baron Quadras mezclaron su patrocinio artístico con el nacionalismo de la Lliga. MACKAY ha captado esta coyuntura:

En aquestes i d'altres obres hom adverteix un cert "aire de familia» - una funció de moda en el context d'una clase social que declarava la seva riquesa recent i la seva identitat nacional - i també l'evidència de la necesitat que cada client, i cada arquitecte, havia d'afirmar la seva individualitat. El gran nombre d'arquitectes disponibles va permetre a la burguesia de complaure's amb unes "despeses notables", legitimades tant per l'individualisme com pel patriotisme. No obstant això, era inevitable que s'havien anat accumulant $i$ que ja es deixaven sentir passessin, de manera dramática a ocupar un primer pla. Les conseqúencies políti-

9 Aunque era una época importante de representación urbana; por ejemplo, en la construcción de la universidad de Barcelona (CASSASAS YMBERT 1988), el Liceu (McDONOGH 1986), etc. 
ques de la nova direcció que prengué el nacionalisme catalá a principis del segle $x x$ tindrien fortes repercussions en l'ambient cultural, $i$ provocaren la crisi del Modernisme en que semblava que aquest gaudia d'una embranzida consistent (1939:63).

El espíritu de la Barcelona modernista, arquitectos y patrones, cedía paso a principios del siglo al Noucentisme, con sus valores "neo-mediterráneos" que contenían una revalorización de la identidad catalana dentro de la ciudad ordenada.

Fou en aquells moments que la problemática social esclata de forma violenta, mentre la burgesia catalana, comoguda pel sotrac, s'inhibia, i l'order públic - conseqúencia de la política de Prat tal i como la predicava Ors- es va posar en práctica en la mesura que no permitia Madrid. Es aquest el marc en què es van canalitzar les aspiracions nacionalistes com també la recerca de la identitat en l'autoritat de les institutions. En aquest sentit, la concepció de la ciudad va ser una fugida de la realitat ...Després del 1917 s'havia d'afrontar la crisi. Mentre, cada cop es feia més forta una identitat collectiva basáda en el "seny" - la imatge tradicional que els catalans tenen d'ells mateixos, una barreja de ponderació mental, mesura i pragmatisme - i encarnada en les institutions de la Mancomunitat (1989:76-77).

Vemos, pues, un retroceso importante en el papel de la élite como mecenas. Los edificios de los grandes arquitectos modernistas y noucentistas de los años 1910-1931 solían ser de construcción pública, salvo en zonas rurales. En la carrera de Antoni Gaudí, un arquitecto que antes funcionaba bajo un patrocinio casi medieval, también se nota un cambio marcado por la terminación -disputada- de la Casa Milà (1911) y el abandono del Parc Güell (1914) ${ }^{10}$.

En las construcciones urbanas antes de la Exposición, se manifiesta otra estrategia hegemónica. Josep Goday Casals, un arquitecto importante de escuelas entre 1918 y 1932, se preocupaba de la educación social y la formación moral de los estudiantes. Sus obras maestras se ubicaron con frecuencia en barrios marginados: dos en el Raval y otra en la Barceloneta (Goday 1932; MACKAY 1989: 80-84, 108) ${ }^{11}$. El mismo arquitecto

1o Rojo Albarrán (1986, 1988) ha propuesto una teoría que explica el abandono del Parc Güell a causa de la crisis del nacionalismo catalán dentro de su potencial clientela burguesa. Veáse Rafols 1952; Bohigas 1968; Collins y Bassegoda 1983; Mackay 1989.

1 El Raval es una zona portuaria cuya sección al lado del puerto, el barrio chino, tenía fama como zona de prostitución y mala vida (McDONOGH 1987). La Barceloneta es otra zona de trabajadores del puerto y pescadores. 
construyó el pabellón de la ciudad de Barcelona de la Exposición de 1929 (Grandas 1988).

Los años de Primo de Rivera fueron caracterizados por MACKAy por un «sentiment d'inquietud generacional enfront de l'incertesa dels temps, una desorientació que compartia la generació anterior que s'havia vist exclosa de l'empresa noucentista" (1989:87). La inquietud evidente en el capital económico y social se realizó en una inquietud de representación arquitectónica. En este ambiente, algunos arquitectos, incluso un descendiente rebelde de la familia López, Josep Lluís Sert, siguieron las ideas modernas de Le Corbusier que llegarían a su culminación en la formación del GATCPAC/GATEPAC y su influencia dentro de la Segunda República (FrEIXÁ 1979; MACKAY 1989).

Pero la simetría importante para nuestro retrato de la élite antigua se cierra con la Exposición retrasada de 1929. A pesar de sus efectos urbanos y de algunas obras maestras como el pabellón alemán de Mies Van der Rohe, "la distància qui hi havia entre l'arquitectura catalana i el moviment modern europeu... quedá immillorablement demostrada en les construcciones de l'Exposició Internacional del 1929" (MACKAY 1989: 90). María Carmen Grandas, que ha publicado un estudio importante de la Exposición, nota:

Amb la celebració de l'Exposició Internacional de Barcelona es posá en evidència la crisi existent en el camp arquitectònic. El canvi de criteris esdevingut de la situació política espanyola des de 1923 provocà que els arquitectes recerquessin en el passat tipologies i llenguatges que els permetés de trobar una solució adequada als projectes que se'ls va encomanar. L'arquitectura de l'Exposició manifesta un historicisme que s'ha de qualificar en sentit peioratiu d'eclèctic, ja que en ella s'ofereixen els més variats discursos estilistics que es mesclen en un mateix edifici... El resultat aconseguit va a ser el de una arquitectura "teatral» i perecedora... (1988:23-4).

A pesar de la posibilidad de una crítica alternativa de algunos edificios, se nota aquí una falta de mensaje, de representación de la ciudad y de sus élites ${ }^{12}$.

\footnotetext{
12 Mackay contrasta el pabellón de Mies Van der Rohe con «la monumentalitat feixuga de Palau Nacional... Fins it tot, la severitat de l'adjacent Palau Alfons XIII, de Puig, és pesada. Al Palau de l'Agricultura, de Manuel Mayol (1899-1929) i J.M. Ribas (1899-1959), i, en menor grau, al Palau de les Arts Grafiques, de Durán Reynals i Pelai Martínez (1898) hom pot veure la decadencia absoluta del mediterranisme. Tan sols un parell dels edificis projectats a casa nostra exhibiren un coneixement de les tendencies europees: el pavelló dels Artistes Reunits (avuis enderrocat)... i una sobria contrucció en maó, el paveltó de la Ciutat de Barcelona, de Josep Goday, que mostra una influencia de Amsterdam" (1989: 90). Carmen Grandas ofrece unas evaluaciones más detalladas y más moderadas de todos estos edificios.
} 


\section{CONCLUSIONES: REFLEXIONES SOBRE UNA CASA ENTRE ESPEJOS}

Con referencia a la burguesía industrial de la época pre-revolucionaria, Miguel IZARD ha escrito que "La interacción entre la debilidad y la subordinación de estas burguesías catalanas explicaría, sin duda alguna, sus reacciones frente a otros grupos sociales, o su actitud ante la cuestión nacional o ante la política estatal» (1979: 221). A pesar de los cambios y oportunidades de la posterior época alfonsina, hemos visto contradicciones paralelas en una élite más complicada que se había formado por los lazos entre estas burguesías y otras capas sociales locales. Además, he sugerido que esta élite había empezado a reproducirse dentro de un habitus del grupo limitante en vez de un discurso sobre el poder urbano.

Abner COHEN, después de sus trabajos con grupos tan distintos como los comerciantes Hausa de Ibadan y los banqueros de Londres ha insistido que

In modern industrial society, no less than in simple society, symbolic patterns of behavior are as live and as significant as any of the manifestations of order and rationality. We are not always aware of the ubiquity of these patterns because we are immersed in them, because they are part and parcel of our social life and of our very selfhoods. This is why we may learn a great deal about them, or at least identify their existence, when a Nuer or a Tonga anthropologist will carry out field work in our midst (1974: 136).

En el estudio comparativo, Cohen ha descubierto procesos paralelos a los que acabamos de describir en Barcelona entre otras clases dominantes. En su obra posterior, por ejemplo, COHEN ha demostrado cómo la élite «criolla» de Sierra Leone adoptó sus modalidades entre la hegemonía metropolitana y el control local (1981). El modelo de la distinción y la creación del capital cultural que ha preocupado a Bourdieu durante los últimos años complementa este proyecto y mi análisis de la élite alfonsina de Barcelona. A través de estos dos teóricos, podemos empezar a comprender de nuevo las élites y su comentario sobre el poder en la España del siglo $X X$ como procesos más generales de la dominación en el mundo moderno.

Esto no implica que los cambios de la época alfonsina que hemos visto aquí carecieron de impactos más complicados en Catalunya como entre otras élites regionales. Los cambios políticos y económicos resonaron en todos los sectores de la sociedad catalana y en los conflictos que unían y distinguían a la ciudad como un campo cultural de combate. Sin embargo, urge agregar el análisis cultural a cualquier investigación socio-histórica. Tanto el proletariado como las élites intentaban interpretar 
y aprovecharse de cambios internacionales y estatales; mientras tanto los poderosos negociaban sus relaciones con el estado y sus grupos de influencia.

Pero cambios importantes entrelazaban siempre con las estructuras y mentalidades de contradicción. Tal vez debemos añadir otro espejo, el de la historia. Empecé con dos pasajes de La Veu de Catalunya, órgano del nacionalismo catalán burgués de la Lliga, que a pesar de veinte años de distancia, mostraron la misma interpretación de su contexto político, las mismas sospechas de los motivos de centralistas y obreros. No cae fuera de las sospechas $e$ interpretaciones que han surgido en las campañas políticas de los 70 y los 80 . La cadena de monumentos urbanos entre 1888 y 1929 tiene otro lazo de representación y mito con los juegos olímpicos de $1992-y$ hemos oído las mismas preguntas-. Podemos sugerir que los patrones descritos no brotan ni de las élites ni de su época sino de las contradicciones fundamentales de Catalunya y otras nacionalidades dentro de la España contemporánea -unas contradicciones que no se podían resolver con un juego de espejos-.

\section{BIBLIOGRAFIA}

AJUNTAMENT DE BARCELONA 1985: Inicis de la urbanistica municipal de Barcelona. Barcelona.

BARCELONA Y SUS EXPOSICIONES 1929: Barcelona: Suplemento a las noticias.

BoHIGAS, Oriol 1968: Reseña y catálogo de la arquitectura modernista. 2 Vol. Barcelona, Lumen.

- Lluís Domenech i Muntaner 1973: Barcelona, Lluís Carulla Canals.

Bourdieu, Pierre 1972: Outline of a Theory of Practice. Cambridge, At the University Press.

-: 1977: Distinction: Critique du jugement social: Paris, Minuit.

CAPEL SÁEZ, Horacio 1975: Capitalismo y morfologia urbana en España. Barcelona, Los llibres de la frontera. Cartilla Moderna de la Urbanidad. 1928. Barcelona.

CASASSAS YMBERT, Jordi 1988: Elies Rogent i la Universitat de Barcelona. Barcelona, Generalitat de Catalunya, Direccio General d'Arquitectura i Habitatge.

COHEN, Abner 1969: Custom and Politics in Urban Africa. Berkeley, University of California Press 1974: Two-Dimensional Man: An Essay on the Anthropology of Power and Symbolism in Complex Society. Berkeley, University of California Press

-: 1981: The Politics of Elite Culture: Explorations in the Dramaturgy of Power in a Modern African Society. Berkeley, University of California Press.

Colegio Oficial de Arouitectos de Madrid. 1981. Lluis Doménech i Muntaner. Madrid: COAM.

Collins, George R. y BAssegoda, J. 1983: The Designs and Drawings of Antonio Gaudi. Princeton, Princeton University Press.

Culla I ClarA, Joan B. 1986: El Republicanisme Lerrouxista a Catalunya. Barcelona, Curial.

DiGiacomo, Susan 1987: "La Caseta i l'Hortet": Rural Imagery in Catalan Urban Politics", Anthropological Quarterly 60: 4 (October 1987): 160-167.

D’Ors, Eugeni 1906: La Ben Plantada. Barcelona. Espiritu y Fuerza de la industria textil catalana. 1945. Barcelona.

FreIXA, Jaume 1979: Josep Lluis Sert. Barcelona, Gustavo Gili.

Godar Casals, Josep 1932: De Pedagogia politica: Els edificis escolars. Congrés d'Arquitectes de llengua catalana. Barcelona, Primavera 1932.

Gramsci, Antonio 1971: Selections from the Prison Notebooks. New York, International Publishers. 
Grandas, Maria Carmen 1988: L'Exposicio International de Barcelona de 1929. Barcelona, Els llibres de la frontera.

Grau, Ramón, director, La Exposició Universal de Barcelona de 1988. Barcelona, L'Avenç.

GüELl, Xavier 1986: Antoni Gaudí. Barcelona, Gustavo Gili.

Hereu Payet, Pere 1988: Arquitectura i ciutat a l'Exposició Universal de Barcelona 1888. Barcelona, Universitat Politécnica de Catalunya.

IZARD. Miquel 1973: Industrialización y obrerismo: las tres clases de vapor, 1869-1913. Barcelona, Ariel.

- 1979: Manufactureros, industriales y revolucionarios. Barcelona, Crítica.

MACKAY, David 1989: L'arquitectura moderna a Barcelona (1854-1939). Barcelona, Edicions 62.

McDonogh, Gary 1986: Good Families of Barcelona: A Social History of Power in the Industrial City.

- 1987: "The Geography of Evil: Barcelona's barrio Chino", Anthropological Quarterly 60: 4 (October 1987): 174-185.

- En prep. "Culture and Categorization in a Turn-of-the-century Barcelona elite", Cultural Anthropology.

Marfany, Josep-Lluís 1878: "El modernisme literari», Historia Salvat de Catalunya V: 269-300.

Martorell Portas. Vicente; Florens a Ferrer, Alfonso and Martorell Otzet 1970: Historia del Urbanismo en Barcelona: Del Pla Cerdá al Area Metropolitana. Barcelona, Comisiones de urbanismo y servicios comunes de Barcelona y otros municipios.

Mendoza. Cristina y Eduardo 1989: Barcelona Modernista. Barcelona, Planeta.

Mouffe. Chantal 1979: Hegemony and Ideology in Gramsci. In Chantal Mouffe, ed. Gramsci and Marxist Thought. London; Routledge \& Kegan Paul, Ltd., 168-204.

- 1988. Hegemony and New Political Subjects: Towards a New Concept of Democracy. In Nelson, C. and Grossberg, L., Marxism and the Interpretation of Culture. Urbana, University of Illinois Press: 89-102.

PABÓN, Jesús 1952-1969: Cambo. Barcelona, Alpha.

Quaderns D'arquitectura I Urbanism e 1988-9: Josep Maria Jujol, Arquitecte, 1879-1949. 179 180. Octubre, novembre, desembre 1988, Gener febrer marc 1989.

Rafols, J. F. 1952: Antoni Gaudi. Barcelona, Aedos.

Rojo Albarran, Eduardo 1986: Antoni Gaudi, aquest deconegut: El Park Güell. Barcelona, Els llibres de la frontera.

- 1988: Antonio Gaudi ese incomprendido: La cripta de la colonia Guell. Barcelona, Los libros de la frontera.

Rovira Gimeno, Josep M. 1987: La arquitectura catalana de la modernidad. Barcelona, Universitat politecnica de Catalunya.

Rubió Tuduri, Nicolau Maria 1927: Dialegs sobre l'arquitectura. Barcelona, Imprenta Altés.

- 1933: La caseta i l'hortet. Inhabilitació de les cases mass velles. Un programa de acció: La mobilització de la propietat urbana.

- 1935: Politica de l'habitació. II Congres d'Arquitectes de llengua catalana. Tarragona 12-16 Oct. 1935.

RussinYol, Santiago 1978: L'Auca del Senyor Esteve. Barcelona, Selecta. First published 1907.

SOLA-MORALES, Ignasi 1976: “L'Exposició international de Barcelona (1914-1929) com a instrument de política urbana", "Recerques VI: 137-148.

- 1980: Eclecticismo y vanguardia: El caso de la Arquitectura Moderna en Catalunya. Barcelona, Gustavo Gilo.

TerRADES, Ignasi 1978: "The Industrial Colonies: A Test Case for the Relationship between Politics and Economics in Industrial Capitalism", Critique of Anthropology III (12): 39-57.

- 1979: Les colonies industrials: un estudi entorn del cas de l'Ametlla de Merola. Barcelona, Laia.

TORRES I CAPEL, Manuel de 1987: El planejament urbà i la crisi de 1917 a Barcelona. Barcelona, Universitat Politecnica de Catalunya.

VICENS VIVES. Jaume 1958: Industrials i politics del segle XIX. Barcelona, Vicens Vives.

Wright, E.O. 1978: Class, Crisis and the State. London, New Left Books. 